\title{
IS BEJEL SYPHILIS?
}

\author{
BY \\ F. AKRAWI \\ Director of the Department for Venereal Diseas ss, Royal College of Medicine, Bagdad, Iraq
}

\section{Introduction}

In Arab countries of the Middle East, especially in Iraq, Syria, and Palestine, there exists a disease known as bejel, which is similar to syphilis in most of its symptoms and clinical course but which differs from it in certain respects. The disease is very widely distributed among the nomadic and seminomadic tribes, sometimes to such an extent that 90 or even 100 per cent. of the members of one tribe are affected. The first impression gained by any observer is that the disease, with its onset in childhood and often very early childhood, must be transmitted in a non-venereal manner; for the most striking fact about bejel is the complete absence of the primary sore. The disease seems to begin with signs that belong to the secondary stage of syphilis, especially the mucous patch which is the predominant feature of this stage. It is also commonly observed that destructive gummata in the region of the palate and nose are the main and predominant signs of its tertiary stage. Most authors have reported the rarity or even absence of neuro-visceral complications. However, this point needs to be more fully investigated.

These difficulties have raised the question whether bejel is syphilis or not. Hudson (1937), working among the tribes of Deir-el-Zor (boundary region of the Euphrates between Syria and Iraq), considers that "bejel occupies in many respects an intermediate position between syphilis and yaws" and advances the view that "bejel is the archetype of syphilis," meaning that bejel today is nothing but a picture of syphilis in ancient times. He bases his assertions on a long and extensive clinical experience.

Widad (1936), of Basrah, goes so far as to claim that bejel is nothing but yaws. Hasselman (1938), basing his work on that of other physicians in Iraq and on good histological studies, rejects Widad's opinion and thinks that bejel is a common form of syphilis influenced in its course by certain loimological factors. However Hasselman does not try to explain the differences alluded to above.
Iraq has a population of about five million, three of which live under nomadic or semi-nomadic conditions. Field work on bejel done in different parts of the country show that in infected tribes the proportion of infected persons varies between 50 and 100 per cent. Recent studies made by us in one tribe of the Ramadi Liwa showed that 79 per cent. of the tribesmen have the disease in one form or another. From these and other studies it seems to us that the lowest estimation of the number of persons affected by bejel in Iraq cannot be put at less than one million. It is clear, then, that bejel constitutes a problem of major importance to the Iraqi health authorities, who say that if bejel were to be, as Hasselman affirms, a usual form of syphilis, then the cost of treatment, carried out on the same lines as the treatment of European syphilis, would reach the colossal figure of three to four million pounds per year. On the other hand, if the disease is found to be a mild form of syphilis, as is suggested by Hudson, the treatment will be completely different.

It is such considerations as these that induced us to try and solve the problem of bejel. The problem resolves itself into three main questions :

1. Is bejel syphilis?

2. What is the method of transmission?

3. Why does bejel, if it is syphilis, take in some respects a different course from the latter?

Our clinical studies of bejel brought us new facts, but we were unable to answer any of the above questions. We came to the conclusion that it should be possible by experiments to reach the solution.

\section{Observations among the Tribes}

General Observations.-Among the Bedouin tribes, where bejel is prevalent, hygiene is primitive or non-existent. The people arè promiscuous; they live in tents, sleeping mostly on the ground either without or with very little bedding, and 
having no protection whatever against all sorts of insects and especially flies, which usually abound. Their food is generally símple but coarse. It consists mainly of barley bread, milk, and dried dates. The inhabitants of one tent eat from the same vessel and drink milk and water from a common bowl. Children from different tents often drink water from a single vessel and pass it from mouth to mouth in quick succession. One commonly sees children playing around with a terrific number of flies about them, their faces often literally covered with these insects, especially round the eyes, nose, and mouth ; and they make little effort to chase them away. Flies go in and out of the buccal cavity and run along the lips without these children trying to repel them. The same picture is frequently seen in children during their sleep on hot days.

Thus it will be seen that the skin of the Bedouin is constantly subjected to climatic changes and exposed to injuries by insects-mainly flies, fleas, and lice. Injuries may also be sustained at work or from going about barefoot and scantily clad.

It does not seem to have been mentioned by previous authors that the Bedouins frequently have whitish-grey patches on the mucous membranes of the lips and cheeks, and that these disappear almost at once after treatment with vitamin $\mathbf{B}_{2}{ }^{*}$ One also frequently sees fissures at the angles of the mouth, evidently of streptococcal nature.

Clinical Course.-On the whole our clinical observations coincide with those of Hudson (1937) and other observers, so we will limit ourselves here to facts that have not already been pointed out or that have not been sufficiently emphasized.

Clinical observation of bejel in Iraq gives the impression that the disease is much more frequent during the warm season than at other times of the year. However, we do not propose to draw any conclusion from this, because of the difficulties of communication that exist during the cold and rainy season for the poor section of the Iraqi population.

As Lacapère (1923), and Hudson (1937) have pointed out, the disease usually starts in early childhood, contamination being mostly non-venereal. As with yaws, it appears that if bejel once enters a tent it soon involves all the occupants. Moreover, the Bedouins always declare to the investigator that they consider bejel to be like measles, a disease which all children must have at some time or other, and if they escape the infection in early life they frequently have it when they are adults.

The chancre or primary sore has never been

* This observation has been confirmed by E. Hoff. (Personal communication.) observed by us ; neither has it been observed by the many Iraqi physicians posted in outlying stations with whom we carried out a detailed investigation about this surprising fact.

The striking fact about the secondary stage (the "first stage" of bejel) is the predominance of the mucous patch. Very often it is the only sign observed in this septicæmic stage of the disease. In outlying dispensaries the mucous patch is the commonest sign and is seen daily by the physicians.

The other secondary lesions observed by us are : macular roseola, papular and papulo-squamous lesions, erosive papules, annular and circinate lesions, pigmented, impetiginous, ecthymatous, and psoriasiform lesions, and paronychia. Osteo-periostitis is rather common. Probably on the rather dark skin of the Bedouin macular roseola often escapes observation. We have frequently diagnosed the disease through nocturnal pains, mainly in the lower extremities.

In the tertiary stage gummata and-nodular lesions dominate the clinical picture. Gummata of the nose and especially of the soft palate are surprisingly frequent, and can be very destructive, creating gangosa-like pictures. Goundou or goundou-like lesions have never been observed by us. It is interesting to note in connexion with nodular lesions, which ultimately involve a large part of the integument, that the Bedouin will point out that his lesions, like trees, "blossom" again in the spring.

Three cases of vitiligo with positive Wassermann reactions have been observed by us in a group of 170 patients examined in three visits in one district.

Hudson (1935) found that juxta-auricular nodes are not rare in Bedouins, and reports an incidence of $2 \cdot 1$ per cent. in an "unselected consecutive series of 236 adult males." In spite of persistent search we have not been able to find one case among the tribes or in the out-patient department of the Royal Hospital, Bagdad. In three cases of nodules on the legs observed by us during the last fifteen years, the pathologist returned a diagnosis of sarcoids of the Darier-Roussy type. Widad (1936) working in Southern Iraq, reported one case on the elbows, buttocks, and knees.

Nervous Manifestations.-Great emphasis is laid by almost all authors on the fact that bejel or other forms of exotic syphilis either do not cause complications in the nervous system or do so very rarely indeed. However, Hoff and Shaby (1940), working in the city of Bagdad, state : "In our experience we have seen many admissions to hospital from the nomad population for various nervous syndromes which on investigation have proved to be syphilitic." 
They describe three cases of meningo-vascular syphilis in tribal patients who stated that they had had bejel (mucous patches) as the first symptom. They summarize their opinion as follows: "We see many cases of meningo-vascular syphilis among Bedouin Arabs, and if we are to call their type of syphilitic infection bejel, then we must state that manifestations of meningo-vascular involvement due to this form of syphilis are quite common."

Before the publication of his article I was given the opportunity by Professor Hoff to examine his three cases. Subsequently Dr. Shaby showed me several other cases, and with his permission I give below the history of two similar cases observed by him recently at the Neurology and Psychiatry Department of the Royal College of Medicine, Bagdad.

Case 1.-A male (aged 40) from Ramadi was admitted to hospital on Jan. 16, 1949, complaining of headache and blurring of vision for one month. A thorough investigation did not reveal any important previous illness. He gave no history of chancre on the genitalia, but admitted to having had bejel in childhood. A physical examination except for the presence of bilateral papillodema, did not reveal anything of pathological importance. The Wassermann reaction was strongly positive in the blood and cerebrospinal fluid, the latter having a total protein content of $60 \mathrm{mg}$. per $100 \mathrm{ml}$., a trace of globulin, and sixteen lymphocytes per c.mm. He was diagnosed as having cerebral syphilis, probably following bejel.

Case 2.-A boy (aged 16) from Karbala was admitted to hospital on Jan. 8, 1949, complaining of weàkness in the upper extremities with a sensation of numbness in. the hands and feet for the previous three weeks. A thorough investigation did not reveal any stigmata of congenital or acquired syphilis. However, he admitted that his parents had told him he had had bejel in childhood. He had three brothers and two sisters, who were all healthy. Examination revealed claw hands with moderate muscle wasting in the upper limbs. There was some diminution in the muscular power with superficial sensory loss in the hands. The Wassermann reaction was strongly positive in the blood and positive in the cerebrospinal fluid, the latter showing a total protein content of $65 \mathrm{mg}$. per cent., with a trace of globulin and seven lymphocytes per c.mm. The diagnosis was pachymeningitis cervicalis hypertrophica (probably following bejel).

It appears from the above that bejel causes meningo-vascular lesions in the same way that syphilis does. In a personal communication Hoff states : "In the same period of observation of the three cases of meningo-vascular syphilis related in my article with J. A. Shaby, we had under our care nine cases of meningo-vascular syphilis in townsmen. It is noteworthy that the ratio between townsmen and settled farmers on the one hand, nomads or semi-nomads on the other hand, in our clinic is three to one, that is, the same ratio existing between three bejel and nine syphilis cases of the cerebrospinal system." This statement is in opposition to Macqueen's (1934) and Hudson's (1937), who emphasize the rarity of the cerebrospinal complications in bejel as compared to syphilis: Hoff's statement implies that bejel causes nervous complications (meningo-vascular) just as frequently as syphilis does.

We are personally convinced from observation that nervous manifestations are not as rare in bejel as other authors have suggested. However, we think that the relative frequency of such symptoms cannot be determined unless extensive investigations among the tribes are made.

As regards parenchymatous complications, we were shown in the Department of Neurology a tribal sheikh with tabes dorsalis who presented a typical picture of the disease, with lightning pains, loss of the patellar reflexes and ankle jerks, and slight ataxia with Romberg's sign, Argyll Robertson pupils, and a positive Wassermann reaction in the blood and cerebrospinal fluid. The patierit said that he had had bejel in early childhood. $\mathrm{He}$ affirmed that he had not been affected with syphilis, but confessed that he was often in town where he visited brothel houses.

To our knowledge not a single authentic case of general paralysis of the insane with a clear ætiology of bejel has been observed. However, we would point out that among Iraqi tribesmen knowledge about psychoses is very limited. The Bedouin, who consults frequently for such different ailments as bejel, malaria, bilharzia, etc., does not consider mental disorders to come within the realm of medicine; believing these disorders to be a work of the "devil," he consults tribal quacks who, by beating the patient, try to drive the devil out of him. Our impression is that psychosis among the Bedouins is in any case rare, and so far as we know no case has been reported that bears any resemblance to general paralysis of the insane.

Cardiovascular Manifestations.-Hudson and Young (1931) say: "Hypertension is virtually unknown in Bedouin patients and cardiovascular syphilis practically absent. In a series of over one thousand adult patients of various ages, the systolic pressure of only seventeen was over $150 \mathrm{~mm}$. of mercury, and in all our experience with bejel, my colleague and I have never found a clinically substantiated case of aortic valvular disease or aneurysm." Our clinical experience seems roughly to coincide with these observations. However, the matter cannot be considered as settled unless field work among the tribes is done on a large scale. 
Unfortunately modern methods of investigation of the cardiovascular system cannot easily be applied on patients living under primitive conditions in desert or semi-desert areas which are quite often distant from civilized centres. Consequently it has not been possible for us to make a serious attempt to tackle the problem, which must be left for future scientific investigations.

Congenital Bejel.-The hereditary transmission of bejel, almost denied by Hudson and Crosley (1936), is to us a reality. Here, of course, dystrophic manifestations constitute the main evidence, because in most cases the disease is acquired in early childhood. Among 218 persons of one tribe examined without selection in the Ramadi district, we found five with typical Hutchinson's teeth and thirty-one with saddle noses. Seventy-three of the 218 were children between the ages of 1 and 15 years. Most of the saddle noses were observed in the latter group. Hudson (1937) reported two or three cases of interstitial keratitis.

Enquiries made in 111 families showed that out of 565 children born to them, 291 had perished up to the date of investigation and 274 were still alive. There were 51 confessed cases of abortion, and we say purposely "confessed" because the Bedouin woman considers it shameful to have a miscarriage and does not consider it an illness.

There seems to us, therefore, little doubt that the congenital transmission of bejel exists. However, we admit that the number of the dystrophic manifestations observed is far from being proportional to the high incidence of bejel in a given community.

We think the facts can be explained on the following lines: It is agreed that bejel is usually acquired in early childhood, unlike syphilis which is acquired after sexual maturity. One would not expect that bejel in an adult Bedouin woman infected, let us say at the age of 5 years, would be as virulent, or capable of being transmitted with as much force and frequency to her offspring, as syphilis would be in a townswoman who had contracted the disease recently and in the early days of her married life.

We find it very difficult to estimate the fertility of the tribes, and think it dangerous to compare their fertility with that of townsmen for obvious reasons. It is likewise very difficult to evaluate the influence of bejel on child mortality, since these tribes are heavily infected with other serious diseases such as malaria, typhoid, the dysenteries, ankylostomiasis, schistosomiasis, etc. One is sometimes inclined to think of cases infected with two, three, or even four of those diseases at the same time as being pathological museums.
Venereal Transmission of Bejel to Tribesmen and its Accidental Transmission to Townsmen.-We have already said that Bedouins who escape the infection in childhood are very often infected later. We were told (but we never observed this fact), that in such cases the infection could sometimes be venereal. We cannot tell how reliable these statements are.

Children of townsmen sometimes acquire bejel, either by living among the tribes (some townsmen still keep up an old habit of sending their children to the tribes to spend a part of their childhood), or through infected Bedouin servants working in the homes of families in the big towns. We observed one child with mucous patches in whom we suspected the infection to have occurred through a very young Bedouin female servant.

We have never known a syphilitic person to contract bejel, nor a person with bejel to contract syphilis, although we believe further investigations should be made.

\section{Inoculation}

Experimental work on monkeys was impossible because they do noc exist in the country and it is impracticable to import them. On the other hand the inconclusive results of experimental work on rabbits carried out by Pearce (1938) on Chinese syphilis discouraged us from carrying an investigation in that direction, at least for the time being.

Numerous experimenters, cited by Ashburn and Craig (1907), have succeeded in infecting human beings with material derived from yaws. According to Sellards and others (1926), volunteers could be infected with yaws and then reinoculated while secondary lesions were present. Williams (1935) statès : "Sellards and Goodpasture and Lacy and Sellards observed that in subjects who had had yaws some months or years previously (some of them having had treatment) reinoculation gave positive results, more often they developed abortive lesion or no lesions at all These experiments indicated that the development of immunity requires a long time." Jahnel and Lange, quoted by Williams (1935), inoculated twenty-five cases of general paralysis of the insane with yaws and obtained negative results in all except one case, in which one abortive lesion resulted.

Leon y Blanco (1939), Oteiza (1946), and these two authors together (1945) inoculated pinta into thirty-four volunteers and reproduced the disease in them. Leon y Blanco, through cross-immunity tests, proved that persons inoculated with pinta can be inoculated with syphilis also.

Consequently, and in view of the great importance attached to the problem of bejel in Iraq, we tried to 
solve the problem by inoculating volunteers including ourselves.

The written consent of each volunteer was obtained after careful explanation of the facts. We are in a position to declare, after sufficient treatment and long observation, that none of the volunteers today shows any sign, either clinical or serological, of the transmitted disease.

Our aim in these inoculations was threefold: (a) to study whether bejel could be successfully inoculated to syphilitic patients ; $(b)$ to study the symptoms that the inoculations would reproduce in non-syphilitic patients; (c) to study possible and probable methods of transmission.

For (a) eight cases of general paralysis of the insane were inoculated by various methods. All the inoculations remained negative. The observation period was extended to seven months.

For (b) ten volunteers (with no history of syphilis and with repeatedly negative serology) were inoculated using as source mucous patches of Bedouin children. All were inoculated on the lower lips after scarification; in eight cases the results were positive, the other two remaining negative both clinically and serologically for a period of six months. In the positive cases a classical erosive sore appeared seventeen to twenty-two days after and at the site of inoculation; later sub-mental adenitis developed. Typical spirochætes were demonstrated by the dark-field method. The Wassermann reaction became strongly positive twenty to twenty-five days after the appearance of the sore. Re-inoculation of one of the positive cases thirty. days after the first positive Wassermann reaction was tried but failed to produce any lesion. In one case the treatment was purposely delayed and a typical macular roseola appeared fifty-nine days after the appearance of the sore.

For (c) seven volunteers with no history of syphilis, and with the Wassermann reaction repeatedly negative, were inoculated. Five cases were inoculated in the following manner: they were given water to drink from a bowl immediately after a Bedouin child with scarified mucous patches of the lips had drunk from it. Three cases remained negative both clinically and serologically for over two years. The other two remained clinically negative but their Wassermann reactions, which were repeatedly negative before the inoculation (done respectively six and eleven times) became positive respectively seventy-five and thirty-four days after the day of inoculation. Both were observed for two more months after the reversal of the Wassermann reaction and before treatment was begun. Although their Wassermann reactions were persistently positive, clinically they remained without symptoms.

Flies (M. domestica), after having been allowed to feed for a few minutes from a scarified mucous patch of the lip of a Bedouin boy, were allowed to settle immediately after on the lips of the remaining two volunteers. One, who was observed for two years, remained both clinically and serologically negative. The other, without showing any symptom at the site of the inoculation or elsewhere, developed seventy-seven days after inoculation a medium positive Wassermann reaction, which turned to strongly positive thirteen days later. He was again inoculated on his lip after scarification, but this time directly from the mucous patch of a bejel patient. For three months he remained clinically without symptoms, and treatment was begun.

From the above it is clear that :

1. It was impossible to inoculate bejel into eight cases of general paralysis of the insane.

2. It was possible to produce the primary sore on the lips by inoculation after scarification from a mucous patch of a bejel case. In most cases there was corresponding adenitis, and in one, observed for a longer period before treatment begun, a typical macular roseola followed. In all the successful cases the Wassermann reaction became positive.

3. Two out of five volunteers in whom experimental transmission was attempted by means of a drinking vessel developed a positive Wassermann reaction but not a primary sore.

4. Similarly, in one of two volunteers in whom transmission was attempted by flies, a positive Wassermann reaction developed without a primary sore. .

5. Even by scarification, transmission failed in some cases.

6. In the successful cases with primary sores the duration of the incubation period was within normal limits.

7. The change of the Wassermann reaction from negative to positive was within usual time limits but was rather retarded in the few successful inoculations through the drinking vessel and the flies.

8. Superinfection, tried in two cases, failed.

9. In spite of the fact that the results mentioned in (3) and (4) above do not permit definite con. clusions because of the small numbers involved, they are of great significance in many aspects of the bejel problem, as will be seen in the following section. 


\section{The Relation of Eejel to Syphilis}

On the basis of studies done by several authors and ourselves, we believe that the identity of bejel and syphilis can be considered as established. We now propose to consider all the factors that lead us to this belief.

Human Inoculations.- On the one hand, the failure to produce infection with bejel in cases of general paralysis of the insane, and on the other the success in producing within normal time limits typical primary sores in many cases, and a roseola in one case, in non-syphilitic persons, is a major if not a conclusive argument in favour of the identity of syphilis and bejel.

Clinical Studies.-The excellent work of Hudson and others, together with fifteen years' clinical observation made by us, shows clearly that bejel follows the same general course as syphilis and produces most of the well-kritown forms of secondary and tertiary lesions of syphilis of the skin and mucous membranes. However, there are certain points of difference which require explanation.

The Absence of the Primary Sore.-It is this puzzling feature of bejel that has made certain observers deny the common ætiology of syphilis and bejel. We believe on scientific grounds that we have established the identity of the two diseases. How can one then explain the absence of the primary sore in bejel?

Hudson (1937) tries to explain it with the hypothesis of a different immunology and that of a congenital allergic hypersensitivity of the skin. However, he soon doubts his own explanation because " yaws seems to have an initial or "mother" lesion; yet it is also a largely untreated and childhood-acquired treponematosis whose immunology one would expect to resemble that of bejel." In other words Hudson (1945, 1946), having in his mind the unitarian view of treponematosis, cannot adopt the above-mentioned hypothesis, namely different immunology and congenital allergic hypersensitivity of the skin, and he thus leaves the matter unsettled.

Three factors, we believe, play a part both in determining the absence of the primary sore and in shaping this variety of syphilis : (1) difference in the virulence of the virus ; (2) difference in the size of the inoculum ; and (3) difference of immunology in the host.

- We do not want to speculate here about the possible existence of a difference in the virulence of the viruses of bejel and syphilis. Animal experiments alone can decide the issue, as it seems to have been decided in the case of yaws and syphilis by the studies of McLeod and Turner (1946). However, like other observers, we have noted that bejel is an attenuated disease as compared with syphilis, and we are under the impression that a weaker strain of virus operates in its case.

The human inoculations described above show that unde conditions of infection similar to those of syphilis, that is, with massive infection such as those carried on the lips after scarification, the primary sore develops. In other words the patients have been put under conditions very similar to those of a person infected with syphilis through intercourse.

On the other hand those inoculations that dealt with transmission through drinking vessels and flies, although small in the number of successful results, tend to show that no primary sore develops when the infection is not massive and is, so to speak, minimal.

We are aware of the success of Magnuson and others (1948) in infecting rabbits with one spirochæte, and we do not contend the fact that experimental infection of the rabbit can occur regardless of the size of the inoculum. On the contrary, we believe that this happens daily in natural infections among the tribes.

However, we believe that in our inoculations the possibility of a strain of virus (bejel virus) weaker than the Nichol strain (used by Magnuson and his collaborators), should be considered. One must also remember that in our case the method of inoculation differs from that used by these authors.

Furthermore, they show that the transmission of the infection in this manner is not easily effected. We think that the prevalence of the infection among children in a given tribal community can only be explained by the repeated minimal infections that occur daily in the life of these children. It seems very likely that extragenital syphilitic infections as we know them are rare accidental massive infections, while in bejel repeated minimal infections are the determining factors in the production of this nonvenereal and extragenital form of the disease.

On the other hand, unlike Hudson, we do not hesitate to accept the hypothesis of a different immunology and the presence of a congenital allergic hypersensitivity of the skin. We think that these factors may also play a big role, not only in determining the absence of the primary sore, but also in determining the whole course of the disease with its variations from the normal course of syphilis. We believe it is dangerous to compare bejel and yaws, because we think we have proved the identity of syphilis and bejel while most authors on clinical, epidemiological, and experimental grounds do not believe in the identity of syphilis and yaws. 
Mucous Patches in Early Bejel and the later Gummata of the Palate.-The predominance of mucous patches in early bejel, and of gummata of the palate in its later stages. is another difference not easily explained. The only explanation that seems plausible to us is the locus minori resistantice in the mucous membranes of the buccal cavity occasioned by three factors (discussed below under the heading of " natural transmission"), namely : the frequent occurrence of white patches from $\mathrm{B}_{2}$-avitaminosis among the Bedouins; the frequent presence in them of buccal streptococcal fissures and rhagades; and the action of climatic extremes on the exposed part of the lips. The hard nature of the food of these Bedouins may, as suggested by Hudson, be another cause.

Absence of Nervous System Involvement.-We have already said that general paralysis of the insane and tabes dorsalis have not been observed among bejel-infected tribesmen, while meningovascular manifestations are, on the contrary, not uncommon. However, we still feel that this question has not beer sufficiently investigated. The reluctance of the Bedouin Arab to seek medical care for mental disorders may partly account for the absence of general paralysis of the insane in observed cases. Moreover, Diefendorf (1923) and others have proved that the lower classes of society are less inclined than the higher to develop parenchymatous syphilis of the nervous system. In the case of the Bedouins we are dealing with the most primitive section of the inhabitants of the Middle East.

Hereditary Transmission.-We think we have demonstrated that bejel, like syphilis, may be congenital (see paragraph above on "congenital bejel"), and we have tried to explain the special problems that it presents in connexion with such transmission.

Bacteriology and Histopathology.-All observers agree that the causative agent of bejel is indistinguishable from that of syphilis in all its morphological aspects. Animal experiments alone can decide whether there exists a difference of virulence between the two.

The excellent histopathological studies of Hasselman (1938) as well as those unpublished of Zahawi and Mills, of the Pathological Institute of Bagdad, prove the identity of the two diseases. We have often sent bejel specimens to the two latter pathologists without mentioning a provisional diagnosis. They invariably reported a " typical histopathological picture of syphilis."

\section{Natural Transmission and the Non-Venereal Nature of Bejel}

In our field-work at Ramadi we found that 94 per cent. of the patients affected with secondary lesions of bejel (mostly mucous patches) were children below the age of 7 years. This is in agreement with the statement of all the authors who have worked on bejel, namely that this disease is mostly acquired in childhood. Here we would point out that pedophylia is unknown among the tribes. We also emphatically reject Hasselman's assertion that sodomy is prevalent among the Bedouins. Hasselman (1938) bases his assertion on the fact that he had been told that the Arabic word " loath" (sometimes used by certain tribes as a synonym of bejel) derives from "loata," which means sodomy in Arabic. He has certainly been misled in this, as the word " loath" is derived from the verb "laoatha," which literally means "to contaminate."

We agree with Hudson (1936) that bejel is a nonvenereal disease, with possible rare exceptions where certain adults are supposedly infected by sexual intercourse during married life if they happen to have escaped the infection in childhood.

What are, then, the common modes of transmission?

It is possible that kissing and fondling between parents and children and among children play a role. But it is a common observation that in infected families the parents are usually affected with tertiary lesions or are in a late latent stage of the disease, while the children show the infective mucous patch. Consequently if kissing plays a role in the transmission of bejel it must be mainly between child and child. This would imply that, in view of the widespread nature of the infection among the tribes, other ways of transmission must be considered.

Studying the hygienic conditions and the habits of the tribal Arab, one notices the habit of drinking water in a common vessel ; this could, especially in the hot season, play a role in the transmission of the spirochætosis we are studying. This fact led us to attempt experimental transmission through the drinking of water by bejel-free persons from a vessel used immediately before by a patient with mucous patches (see "inoculations" above). We succeeded twice in transmitting the disease, and the Wassermann reaction became positive. But; to our surprise, the two patients unexpectedly did not develop any primary lesion in spite of minute daily inspection. The positive Wassermann reaction was the first sign of the disease in them. For years preceding the experiment it had been repeatedly 
negative, and now remained positive until treatment was begun.

Two important facts emerge from this experiment:

1. That the habit of drinking water from a common vessel very likely plays an important role in the transmission of bejel among the tribes. This would be a plausible explanation of the colossal incidence of infection in a given community.

2. That in bejel, as in the rare cases of syphilis without chancre, the primary stage may be omitted. In fact, in the two cases, bejel entered directly into the latent stage, thus falling into the group described by some French authors in syphilis as "syphilis latente d'emblée."

Every observer studying conditions among the tribes notices the fly pest. The camps are teeming with these insects. Often during our study of bejel we saw flies actually entering the buccal cavities of children and then settling on the mouth of the next child. This suggested that the fly may be an important mechanical vector in transmitting the disease. So we were led to the experiment mentioned above in which flies, after settling on a scarified mucous patch, were made to settle for a few minutes on the lip of a nop-syphilitic, bejel-free volunteer (see "inoculation" above). As in the experiment with the drinking vessel, no sore developed, but the Wassermann reaction; which was previously repeatedly negative, became positive about two months later. From this we conclude that flies are likely to play a role in the transmission of bejel. For the sake of comparison we refer the reader to the experimental work of Lamborn (1936) on the transmission of yaws by flies to man.

The question arises why these factors do not play the same role in the transmission of syphilis in townsmen. It will be noted that in spite of the ideal conditions under which our inoculations were carried out, they failed in a number of the cases. However, under natural conditions as we have briefly described them among the tribal population, the frequency of the exposures rarely permits a child to escape infection. This frequency of exposure is rare under the much more hygienic conditions in the town.

We believe that three other minor factors play a - certain role in favouring these modes of transmission :

1. The white patches of $B_{2}$ avitaminosis which are so often encountered on the mucous membranes of the buccal cavity in Bedouins and which are amenable to treatment.
2. The streptococcal fissures and rhagades frequently found on the lips and angles of the mouth of these Bedouins.

3. Desquamation and fissures of the lips caused by the severity of the climate, especially the sun's rays, are very frequent.

\section{Measures to Fight Bejel}

The main aim of this work was to arrive at a practical conclusion which could help the countries concerned in their fight against this endemic disease.

Although we think we have proved the identity of bejel and syphilis, we cannot recommend for bejel the same counter-measures that are usually advised in the case of syphilis. In syphilis, where contamination is mostly venereal, the fight against prostitution and the search for and thorough treatment of the infected persons are the main prophylactic measures. In bejel, apart from the admitted fact that contamination is mostly non-venereal, the tremendously widespread nature of the disease necessitates different methods for dealing with it.

We believe we have sufficiently proved that the non-hygienic living conditions that prevail among the tribes concerned are responsible for the facility with which the disease is transmitted, and we have specially emphasized the fly pest, the common drinking vessel, and $\mathbf{B}_{2}$ avitaminosis. Therefore it is clear that the improvement of the hygienic conditions of these tribes would be the basis for any effective prophylaxis against this disease.

But as we are here dealing with nomadic and seminomadic populations, no improvement in their hygienic conditions can be expected unless a wide programme of land settlement is carried out. This, of course, would be the real key to, and the ideal solution of the problem of bejel. However, in the present circumstances prevailing in Iraq, the greatest difficulties stand in the way of such a solution, and this may be so for some years to come. The same can be said of the treatment of the individual on the same lines as the treatment of syphilis, because, bejel being such a widespread disease (in some localities the incidence of the disease is almost 100 per cent.), in so poor a community as that of the tribes it is evident that no government in the Middle East can bear the expenses of such a campaign, which would have to be assiduously carried out for many years. We will not discuss the thorny problem whether such measures against a seemingly attenuated disease (bejel) would not result ultimately in the prevalence of a more malignant one (syphilis) with far-reaching neuro-visceral complications, because we think there is no tangible and convincing evidence that this would be likely. 
To every observer of bejel it is obvious that the mucous patch is the main source of this widespread infection. It is, therefore, the mucous patch that must be looked for and sterilized as early as possible by anti-syphilitic treatment, to prevent wholesale, widespread infections among children. The treatment, then, need be symptomatic only and for the sole purpose of prophylaxis to stem the spread of the disease. This aim, we believe, can be more or less easily realized under present circumstances. Ambulatory dispensaries, while carrying on the fight against other endemic diseases such as malaria, ankylostomiasis, etc., can look for cases of mucous patches and treat them symptomatically with a few injections of one or more of the anti-syphilitic drugs, such as arsphenamine, bismuth, and even penicillinin-oil and beeswax if that were found to be economical.

We have not had a wide experience of the treatment of bejel with penicillin. We have had the opportunity to treat only six cases in various stages of the disease with sodium penicillin at three-hourly intervals. This work is still under progress but we can already state that bejel responds to penicillin.

\section{Conclusions and Summary}

1. Bejel is a widespread spirochætosis among certain tribes of the Middle East countries. It differs from syphilis mainly in the absence of a primary sore, in the predominance of the mucous patch in the early stages and the gumma of the palate in the later stages, and in its non-venereal mode of infection among children in most cases.

2. The identity of bejel and syphilis is proved by the coinciding clinical symptomatology in the two diseases, the existence of congenital bejel (but with some explainable variation as compared to syphilis), the presence of meningo-vascular manifestations in both diseases, the failure to infect general paralytics with bejel, and the experimental reproduction of a primary sore in non-syphilitic volunteers, and the identity of its bacteriological and histopathological aspects.

3. The difference between the clinical aspects of bejel and that of syphilis are fully discussed. We have tried to explain them on both theoretical and experimental grounds.
4. Transmission through drinking water with a common vessel and through flies (mechanical vectors) is proved. $B_{2}$ avitaminosis, streptococcal rhagades and fissures, and the severity of the climate also probably play a role.

5. In view of the fact that the epidemiology of bejel differs from that of syphilis, the ideal method of attacking the disease is the improvement of the hygienic conditions of the tribes by an extensive land settlement scheme. A temporary measure is proposed whereby the mucous patch, which is the main source of infection, could be dealt with.

We are indebted to Dr. H. Hoff, whose great interest in our work was inspiring to us. We are also indebted to Dr. E. A. Mills for having read the manuscript.

\section{REFERENCES}

Ashburn, P. M., and Craig, C. F. (1907). Phillipine J. Sci., 2, 452 .

Diefendorf, A. T. (1923). "Clinical Psychiatry." Fifth Edition. p. 276. The Macmillan Company, New York.

Hasselman, C. M. (1938). Arch. Derm. Syph., Chicago, $38,837$.

Hoff, H., and Shaby, J. A. (1940). Trans. R. Soc. trop. Med. J,Hyg., 33, 549.

Hudson ' E. H., and Young, A. L. (1931). Amer. J. trop. Med., 11, 297.

Hudson, E. H. (1935). Trans. R. Soc. trop. Med. Hyg., 28, 511 .

- (1936). Arch. Derm. Syph., Chicago, 33, 994. (1937). Trans. R. Soc. trop. Med. Hyg., 31, 9. (1945). Oxford Medicine, V. pt. II, p. 656 (9). -(1946). Amer. J. trop. Med., 26, 135.

_, and Crosley, S. S. (1936). Brit. J. Derm. Syph., 48, 288.

Lacapère, G. (1923). "La Syphilis Arabe.” Octave Doin, Paris.

Lamborn, W. A. (1936). J. trop. Med. Hyg., 39, 235.

Leon y Blanco, F. (1939). Medicina, Mexico, 19, 17.

, and Oteiza, A. (1945). Science, 101, 309.

McLeod, C., and Turner, T. B. (1946). Amer. J. Syph., 30, 442.

Macqueen, J. (1934). Brit. J. vener. Dis., 10, 33.

Magnuson, H. J., Eagle, H., and Fleischman, R. (1948). Amer. J. Syph., 32, 1.

Oteiza, A. (1946). Bol. Soc. cubana Dermatol. Sif., 3, 39.

Pearce, L. (1938). J. exp. Med., 67, 443.

Sellards, A. W., Lacy, G. R., and Schöbl, Phillipine J. Sci., 30, 463.

Widad, M. (1936). "Is Bejel Yaws?" (In Arabic). Government Press, Bagdad, Iraq.

Williams, H. U. (1935). Arch. Path.; 20. 596. 